\section{Dinámica de la superficie agrícola cultivada en Colombia, 1960-2010}

\section{Dynamics of agricultural area cultivated in Colombia, 1960- 2010}

\author{
Fernando Restrepo Betancur, Holmes Rodríguez Espinosa, Marisol Medina Sierra
}

Universidad de Antioquia UdeA.

\section{Resumen}

La inserción de Colombia al mercado global, como consecuencia de la apertura económica ha : generado cambios importantes en su economía con efectos en la estructura agraria del país por lo : cual es importante conocer la dinámica de comportamiento de la superficie cultivada que permita : establecer políticas prospectivas para el desarrollo sectorial. En este sentido el objetivo del estudio : fueanalizar la dinámica de la superficie agrícola cultivada en Colombia en el período 1960-2010. Se : trabajó con información de (Faostat) que fue analizada utilizando análisis descriptivo exploratorio : de tipo unidimensional, análisis comparativos mediante la técnica multivariada de la varianza : (Manova), análisis de componentes principales y análisis de correlación método de Spearman. : Se encontró una reducción en el área destinada a la agricultura con un incremento del área en : ganadería. Se encontró diferencia altamente significativa $(\mathrm{p}<0.0001)$ en la superficie cultivada para los principales cultivos. Se detectó diferencia altamente significativa $(\mathrm{p}<0.0001)$ en la superficie : cultivada para los cultivos evaluados. Algodón, Maíz y Trigo han tenido un decrecimiento notorio : en la superficie utilizada, caso contrario al cultivo de hortalizas, frutales, papa, plátano, fríjol y caña de azúcar. El contraste canónico, permitió detectar diferencias entre la década de los 90 respecto a : la del 70, 80 y la primera década del siglo XXI. Las políticas públicas implementadas en Colombia : han conducido al sector agrario a una reestructuración que ha acrecentado las diferencias entre la : agricultura con destino a la agroindustria y la exportación y la agricultura campesina con destino al $\vdots$ mercado doméstico.

Palabras clave: Análisis multivariado, desarrollo agrícola, políticas agrarias.

\begin{abstract}
Colombian entrance in the global market as a result of the economic opening, has resulted in : important changes of its economy, with effects on its agricultural structure; therefore, it becomes : important to learn the performance dynamics of the cultivated area, which leads to establish policies : for this sector development. In this sense, the purpose of this study was to analyze the dynamics : of agricultural area cultivated in Colombia, in the term 1960-2010. FAOSTAT information was : used; it was analyzed through a single-dimension exploratory descriptive analysis, comparative : analyses through variance multi-varied technique, MANOV analysis of the main competences, and : correlation analysis Spearman method. A reduction was found in the area destined to agriculture, with an increase in cattle breeding area. A highly meaningful difference $(p<0.0001)$ was found in the cultivated area for the main crops. A highly meaningful difference $(\mathrm{p}<0.0001)$ was detected in :
\end{abstract}

Recibido: 19/02/2016

Revisado: $11 / 05 / 2016$

Aceptado: 10/12/2016

Correspondencia de autor: frbstatistical@yahoo.es holmes.rodriguez@udea.edu. co

(C) 2016 Universidad La Gran Colombia. Este es un artículo de acceso abierto, distribuido bajo los términos de la licencia Creative Commons Attribution License, que permite el uso ilimitado, distribución y reproducción en cualquier medio, siempre que el autor original y la fuente se acrediten.

Cómo citar:

Restrepo, F., Rodríguez, H. Medina,M. Dinámica de la superficie agrícola cultivada en Colombia, 1960-2010. UGCiencia 22, 85-98. 
cultivated area for evaluated crops. Cotton, corn and wheat have increased in the area used; opposite to cultivation of vegetables, fruit, potato, banana, beans, and sugar cane. The canon contrast led to detect a difference among the $90 \mathrm{~s}$ regarding the $70 \mathrm{~s}$, and $80 \mathrm{~s}$, and the first decade of XXI C. Public policies implemented in Colombia have led the agricultural sector to a restructuring, and has increased the difference between agriculture destined to agroindustry and export, and peasant agriculture destined to domestic market.

Key words: Multi-varied analysis, agricultural development, agricultural policies.

\section{Introducción}

El sector agropecuario ha sido uno de los principales motores del desarrollo económico colombiano; aporta cerca del $9 \%$ al PIB, contribuye con el $21 \%$ de las exportaciones y genera el $19 \%$ del empleo total del país (Vélez, Campos, Córdoba y Anzola, 2010). En Colombia, se usan para actividades agrícolas 4.9 millones de hectáreas de las cerca de 21.5 millones que se pueden dedicar a la agricultura; por el contrario, para actividades pecuarias se utilizan 38.6 millones de hectáreas, de las cuales solo 20 millones son aptas para explotaciones ganaderas (Restrepo, 2010).

Los procesos de apertura económica llevados a cabo en Suramérica a finales del siglo XX, como resultado de las políticas de liberalización y desregulación de los mercados, han ocasionado importantes cambios en la estructura del sector agrícola de los países, trayendo consigo dificultades como las desigualdades económicas y el incremento de la migración rural a las zonas urbanas (Baudasse y Calderón, 2009), como consecuencia de la consolidación de esquemas de agricultura comercial orientada a la exportación que generan problemas de empleo rural (Soto, 2003).

En Colombia la producción agrícola se ha venido concentrando en cultivos permanentes, los cuales representan cerca del $60 \%$ del área total; los transitorios por su parte ocupan el 33\% mientras los forestales el 7\% restante (Restrepo, 2010). Esta situación ha ocasionado una división en los sistemas de producción, entre los campesinos que explotan pequeñas parcelas en las laderas de las montañas destinadas principalmente a cultivos de subsistencia, y por otro lado los terratenientes en las tierras más fértiles y accesibles destinadas a cultivos comerciales.

Teniendo en cuenta la nueva realidad colombiana, en un escenario de acuerdos de paz, es importante pensar en las estrategias para el desarrollo del área rural, la más golpeada por la violencia en los últimos 50 años, a partir del análisis de la dinámica de la superficie sembrada en las últimas décadas y la proyección de la producción agrícola para los años venideros. En este sentido, Lowe, Murdoch, Marsden, Munton, y Flynn (1993) reportan que el desarrollo de regiones rurales, depende de factores de tipo económico, social y político; Porter (2007) por su parte, plantea que para lograr el desarrollo, además de la capacidad de un país de inserción al mercado global y de atracción de la inversión extranjera, requiere fortalecer las capacidades básicas competitivas.

Cazalis (1980) plantea que para lograr el desarrollo agrícola se deben tener en cuenta aspectos como el mejoramiento de la eficiencia basada en la especialización productiva y el uso del suelo. Al respecto, las políticas de desarrollo agrícola a nivel global, han procurado resolver problemas de la producción, basadas en la provisión de bienes públicos y desarrollo de 
la infraestructura (Norton, 2004); incentivos a los productores, tenencia de la tierra, acceso a la tecnología agrícola y al crédito, entre otras. Sin embargo, el estado ha venido pasando a una situación menos protagónica en el desarrollo agrícola.

Es importante por lo tanto, analizar el resultado de las políticas públicas en el desarrollo agrícola, teniendo en cuenta el comportamiento de la superficie sembrada en los principales cultivos agrícolas del país. Por lo anterior, el objetivo de este estudio fue evaluar la dinámica de la superficie sembrada en cultivos en Colombia en el período 1960-2010, a partir del análisis multivariado, método utilizado por otros autores para análisis de política agrícola (Delgadillo y Chalita, 2013).

\section{Materiales y métodos}

A partir del objetivo plateado y la información secundaria disponible en la base de datos de la FAO en su portal Faostat, se seleccionaron para el análisis los principales cultivos sembrados en Colombia. En la tabla 1 se presenta la descripción de las variables utilizada en el estudio.

Tabla 1. Descripción de las variables.

\begin{tabular}{lclc}
\hline \multicolumn{1}{c}{ Variable } & \multicolumn{1}{c}{ Tipo } & \multicolumn{1}{c}{ Variable } & \multicolumn{1}{c}{ Tipo } \\
\hline Década & Cualitativa & Superficie en maíz & Cuantitativa \\
Superficie agrícola total & Cuantitativa & Superficie en caña & Cuantitativa \\
Superficie en cacao & Cuantitativa & Superficie en arroz & Cuantitativa \\
Superficie en yuca & Cuantitativa & Superficie en café & Cuantitativa \\
Superficie en trigo & Cuantitativa & Superficie en frutales & Cuantitativa \\
Superficie en sorgo & Cuantitativa & Superficie en papa & Cuantitativa \\
Superficie en frijol & Cuantitativa & Superficie en hortalizas & Cuantitativa \\
Superficie en pastos & Cuantitativa & Superficie en cereales & Cuantitativa \\
Superficie en plátano & Cuantitativa & Superficie legumbres & Cuantitativa \\
\hline
\end{tabular}

Fuente: elaboración propia
Los datos fueron analizados utilizando análisis multivariado de la varianza -Manova- con contraste canónico de tipo ortogonal mediante el modelo lineal general (GLM), a fin de comparar si existe diferencia entre las décadas para la superficie asociada con los diferentes cultivos. Adicionalmente, se realizó análisis de componentes principales utilizando el método R; se aplicó matriz de correlación por la técnica no paramétrica de Spearman, finalmente, se empleó el análisis descriptivo de tipo exploratorio unidimensional. Para el procesamiento de la información se utilizó el paquete estadístico $S A S$ University Edition Virtual App.

\section{Resultados}

Eluso de la tierra en Colombia ha caído de manera marcada en la última década como se indica en la figura 1. La superficie cultivada de yuca en Colombia tuvo su punto de máxima superficie cultivada a comienzos de la década de los 70 hasta mediados de la misma década, sufriendo un decremento notable a partir del año 1976 hasta 1988, a partir de dicho año se presentó un pequeño incremento, permaneciendo con poca variabilidad hasta finales del año 2010. Para el cultivo de trigo se nota una caída significativa en la superficie cultivada;incrementándose notoriamente la importación de tal cereal, el cual es fundamental para la nutrición de la población.

El cultivo de sorgo sigue un patrón tendiente a una distribución normal; a partir de mediados de la década de los 90 ha caído de manera abrupta. El cultivo del maíz ha tenido un patrón de dinámica de comportamiento muy particular en el sentido de que las caídas en la cantidad de superficie cultivada han seguido el mismo comportamiento a lo largo del ciclo del cultivo. Entre 1975 y 1995, se presentó el 
Figura 1. Dinámica de comportamiento de los principales cultivos
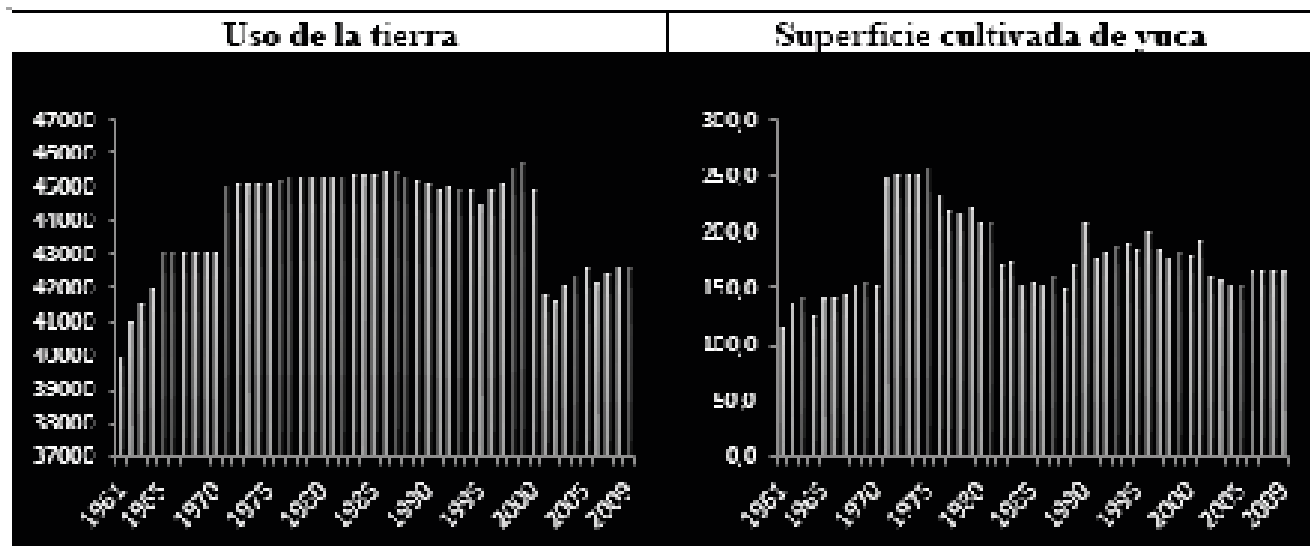

Superficie cultivada de trigo

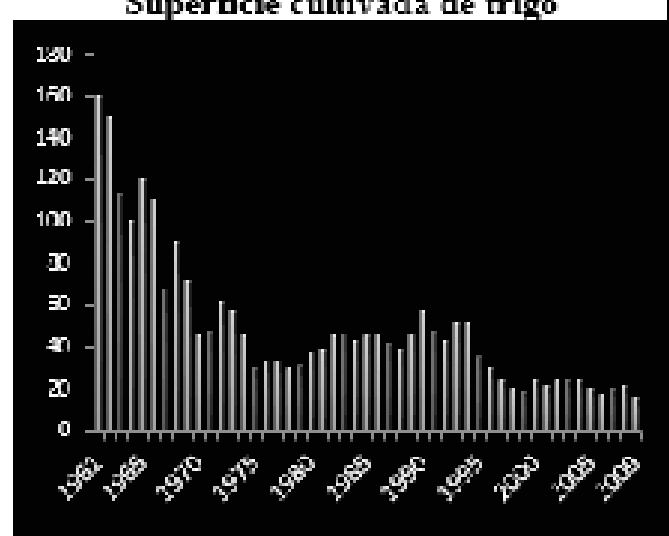

Superficie cultiv ada de frijol

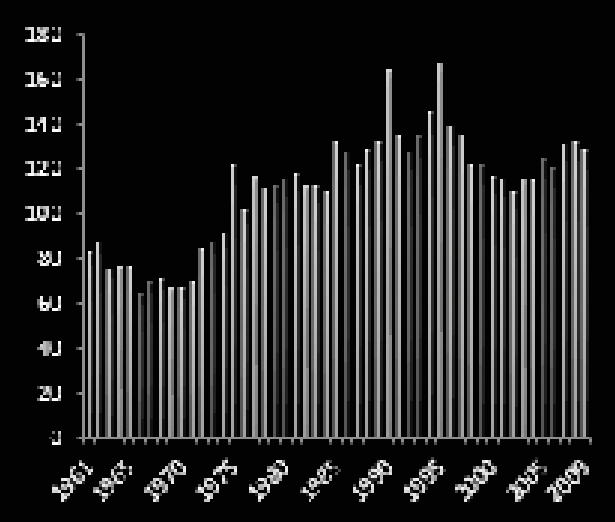

Supcrficic cultivada de sorgo

Superficie cultivada de maiz

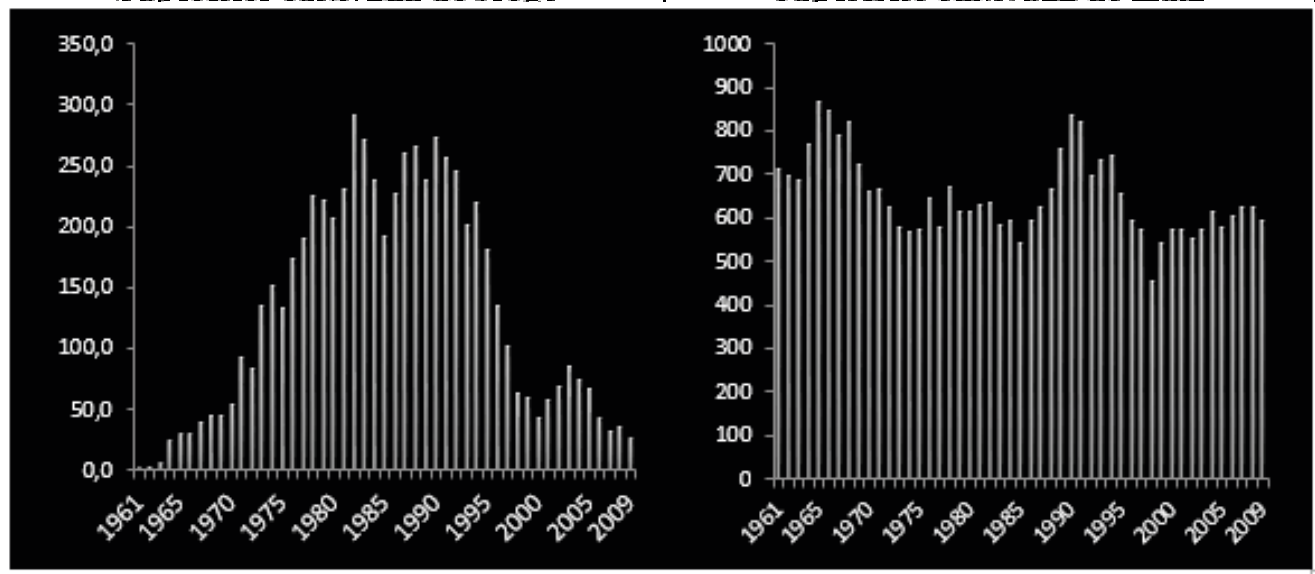


mejor comportamiento relacionado con la superficie cultivada de frijol, decayendo a partir de este período. La superficie sembrada de caña de azúcar ha presentado un incremento casi constante en los últimos quince años. El cultivo del arroz sigue una dinámica con cambios bastante fluctuantes en el tiempo.

En cuanto al cultivo de frutales como se muestra en la figura 2, presenta una tendencia creciente en el período analizado. El cultivo de algodón se ha caracterizado por tener una tendencia alcista entre 1961 y 1980; a partir de dicho año ha decaído de manera apreciable, en relación a la superficie cultivada. Similar dinámica ha tenido el cultivo del cacao, anotando que la tendencia creciente se prolongó hasta 1995.

Figura 2. Evolución de la superficie utilizada en los cultivos de: azúcar, arroz, algodón, legumbres, frutales y cacao.

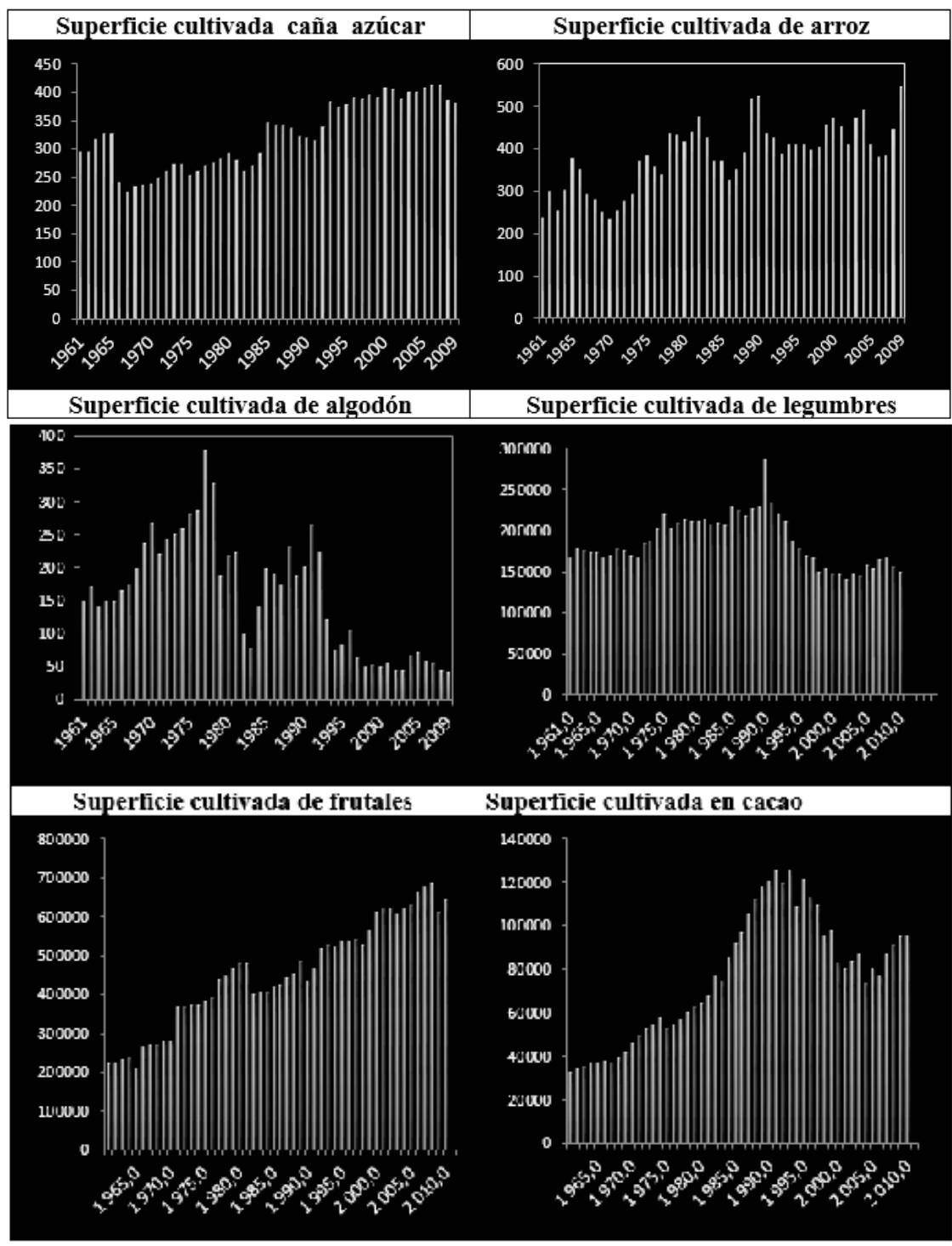


En la dinámica de comportamiento a través del tiempo de los cultivos: café, plátano, hortalizas, papa y cereales, como se muestra en la figura 3, se destaca que para el cultivo de la papa ha existido una caída en la superficie total cultivada a partir del año 2000; el cultivo del plátano ha tenido una dinámica similar de crecimiento desde 1975; las hortalizas tienen dinámica alcista a partir de 1995, mientras los cereales posee dinámica decreciente en términos generales desde 1990.

Figura 3. Evolución de la superficie utilizada en los cultivos de: café, plátano, hortalizas, papa y cereales.

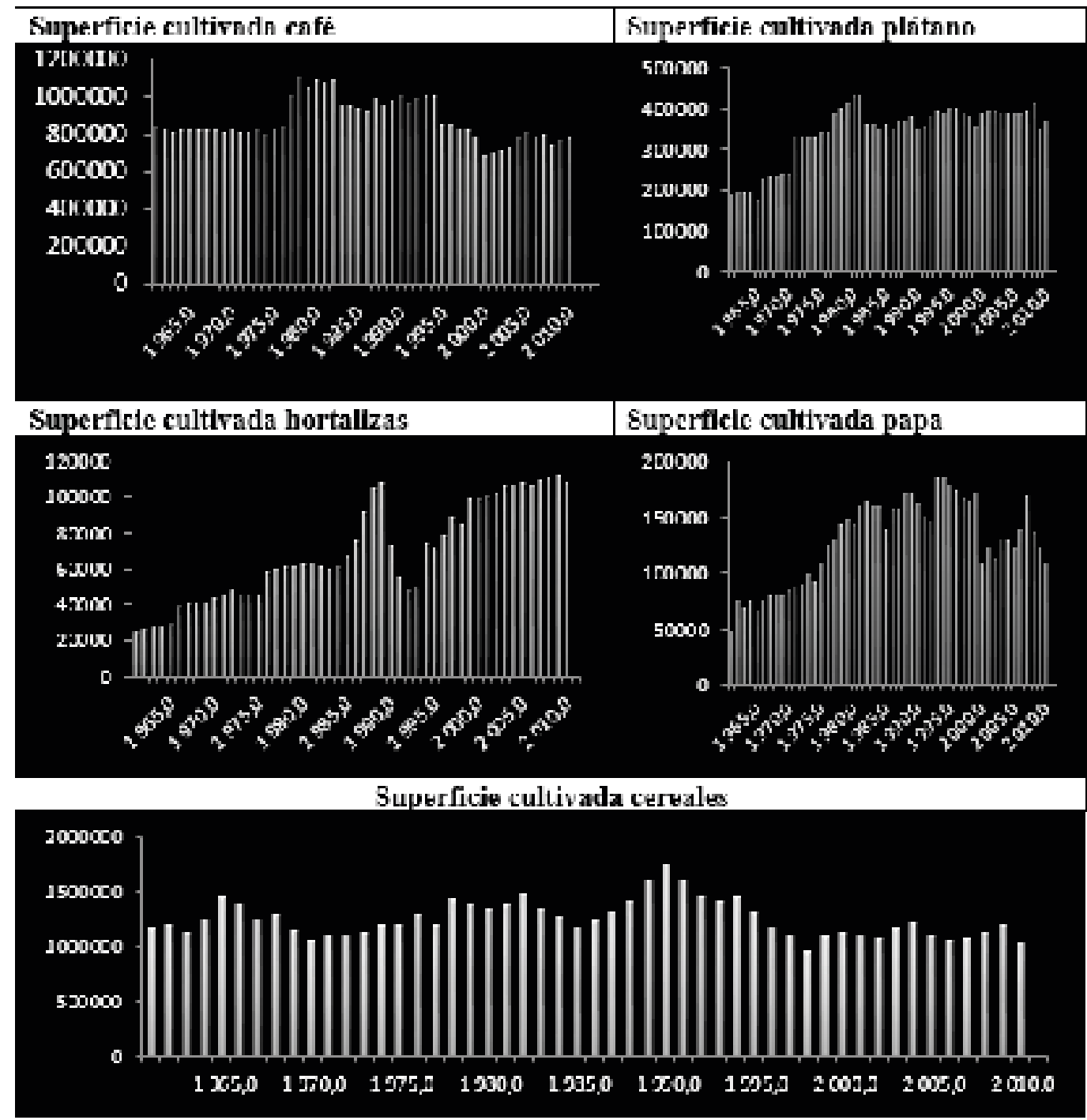


En cuanto al promedio de superficie cultivada expresada en miles de hectáreas, como se observa en la tabla 2 (ver sig pág), se puede destacar como los cultivos de trigo y algodón han caído de manera significativa a través del tiempo, al igual que el cultivo del maíz, por el contrario cultivos como la caña de azúcar, las frutas y el arroz han incrementado la superficie cultivada de manera notoria.

Tabla 2. Promedio de superficie cultivada en miles de hectáreas.

\begin{tabular}{ccccccccc}
\hline Década & Algodón & Arroz & Trigo & Sorgo & Maíz & Caña.Azú. & Fríjol & Yuca \\
\hline $\mathbf{6 0}$ & 170.1 & 292.6 & 109.1 & 25.0 & 768.4 & 276.7 & 73.9 & 139.4 \\
$\mathbf{7 0}$ & 269.6 & 335.9 & 41.1 & 145.9 & 619.0 & 262.9 & 95.8 & 229.6 \\
$\mathbf{8 0}$ & 173.0 & 407.2 & 42.7 & 242.1 & 623.3 & 307.9 & 120.7 & 169.7 \\
$\mathbf{9 0}$ & 122.8 & 424.0 & 37.5 & 173.6 & 664.4 & 365.9 & 138.7 & 185.8 \\
$\mathbf{2 0 0 0}$ & 51.4 & 448.6 & 19.5 & 50.1 & 583.7 & 394.0 & 119.8 & 171.9 \\
\hline Década & Café & Cacao & Hortal & Papa & Plátano & Frutas & Cere ales & Legum. \\
\hline $\mathbf{6 0}$ & 815.4 & 36.9 & 33.0 & 72.1 & 206.7 & 245.8 & 1247.9 & 172.7 \\
$\mathbf{7 0}$ & 889.0 & 54.7 & 51.2 & 110.7 & 342.3 & 388.9 & 1203.9 & 196.5 \\
$\mathbf{8 0}$ & 991.8 & 89.3 & 75.3 & 158.1 & 375.8 & 439.7 & 1355.5 & 217.4 \\
$\mathbf{9 0}$ & 908.1 & 113.7 & 72.1 & 168.4 & 379.1 & 518.5 & 1326.5 & 195.1 \\
$\mathbf{2 0 0 0}$ & 749.9 & 84.8 & 106.2 & 127.2 & 386.9 & 636.1 & 1110.7 & 151.6 \\
\hline
\end{tabular}

Al calcular el coeficiente de variación para cada década asociada con los cultivos citados, en la tabla 3 (ver sig pág) se destaca la gran heterogeneidad para cultivos como el algodón, trigo, sorgo. Cultivos como la yuca, el café y las frutas han tenido una dinámica homogénea a lo largo del tiempo en relación a la superficie empleada para dichos cultivos.

Tabla 3. Coeficientes de variación superficie cultivada

\begin{tabular}{ccccccccc}
\hline Década & Algodón & Arroz & Trigo & Sorgo & Maíz & $\begin{array}{r}\text { Caña de } \\
\text { Azúcar }\end{array}$ & Fríjol & Yuca \\
\hline $\mathbf{6 0}$ & 17.8 & 15.6 & 29.0 & 70.1 & 8.6 & 15.3 & 10.0 & 8.9 \\
$\mathbf{7 0}$ & 19.9 & 21.1 & 27.7 & 39.6 & 6.6 & 5.2 & 20.2 & 13.9 \\
$\mathbf{8 0}$ & 29.4 & 14.3 & 8.2 & 12.5 & 9.3 & 10.5 & 7.0 & 12.7 \\
$\mathbf{9 0}$ & 63.1 & 9.3 & 37.9 & 45.5 & 18.6 & 8.3 & 11.6 & 5.4 \\
$\mathbf{2 0 0 0}$ & 19.4 & 11.4 & 15.2 & 37.8 & 4.2 & 2.8 & 6.4 & 7.2 \\
\hline Década & Café & Cacao & Hortal. & Papa & Plátano & Frutas & Cereales & Legum. \\
\hline $\mathbf{6 0}$ & 0.9 & 6.9 & 21.0 & 13.9 & 11.9 & 10.6 & 8.8 & 2.1 \\
$\mathbf{7 0}$ & 12.8 & 9.3 & 14.9 & 21.5 & 14.3 & 13.4 & 10.2 & 9.3 \\
$\mathbf{8 0}$ & 6.5 & 20.7 & 25.0 & 6.7 & 8.3 & 7.4 & 9.3 & 4.0 \\
$\mathbf{9 0}$ & 9.9 & 9.5 & 23.1 & 7.7 & 4.9 & 7.4 & 18.8 & 21.9 \\
$\mathbf{2 0 0 0}$ & 5.7 & 8.3 & 4.1 & 13.4 & 4.2 & 4.4 & 5.2 & 5.7 \\
\hline
\end{tabular}

El análisis de componentes principales elaborado con base en la información referente a la superficie cultivada para los cultivos asociados a la presente investigación permitió definir de manera clara tres componentes principales, los cuales explican una variabilidad acumulada del $84.7 \%$, como se observa en la tabla 4 . El factor uno se explicó por los cultivos: arroz, azúcar, frijol, cacao, hortalizas, papa, plátano y frutas. El factor dos lo explicó la dinámica de comportamiento 
de los cultivos: sorgo, café, cereal y legumbres. El factor tres lo explicaron los cultivos: trigo, maíz y yuca.

Tabla 4. Análisis de componentes principales

\begin{tabular}{|c|c|c|c|}
\hline Variables & Factor 1 & Factor 2 & Factor 3 \\
\hline \multicolumn{4}{|l|}{ Algodón } \\
\hline \multicolumn{4}{|l|}{ Arroz } \\
\hline \multicolumn{4}{|l|}{ Trigo } \\
\hline \multicolumn{4}{|l|}{ Sorgo } \\
\hline \multicolumn{4}{|l|}{ Maíz } \\
\hline \multicolumn{4}{|c|}{ Caña de azúcar } \\
\hline \multicolumn{4}{|l|}{ Frijol } \\
\hline \multicolumn{4}{|l|}{ Yuca } \\
\hline \multicolumn{4}{|l|}{ Café } \\
\hline \multicolumn{4}{|l|}{ Cacao } \\
\hline \multicolumn{4}{|l|}{ Hortalizas } \\
\hline \multicolumn{4}{|l|}{ Papa } \\
\hline \multicolumn{4}{|l|}{ Plátano } \\
\hline \multicolumn{4}{|l|}{ Frutas } \\
\hline \multicolumn{4}{|l|}{ Cereales } \\
\hline \multicolumn{4}{|l|}{ Legumbres } \\
\hline $\begin{array}{l}\text { Porcentaje } \\
\text { acumulado }\end{array}$ & 44.8 & 73.2 & 84.7 \\
\hline
\end{tabular}

En el plano factorial, como se observa en la figura 4, se muestra de manera clara una relación entre cultivos, dado por la dinámica del clúster los cultivos azúcar, frutas y hortalizas están en un mismo grupo, cercano al otro clúster conformado por: plátano, cacao, papa, arroz, frijol; otro grupo lo conforman los cultivos de: algodón, maíz y trigo .

Figura 4. Plano factorial

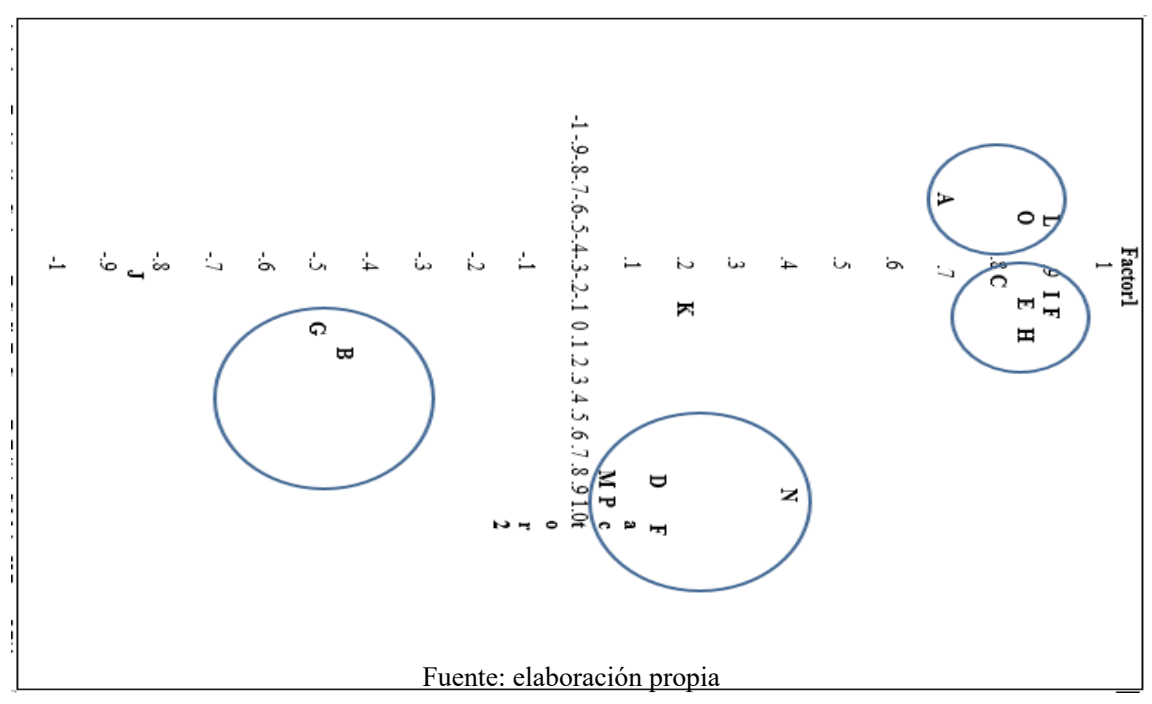


El análisis multivariado de la varianza, como se muestra en la tabla 5, permitió detectar diferencias altamente significativas $(\mathrm{p}<0.0001)$ en la superficie cultivada para los cultivos evaluados.

Tabla 5. Análisis multivariado de la varianza, superficie cultivada. Fuente: elaboración propia, 2015. Letras distintas indican diferencia significativa $(\mathrm{p}<0.05)$

\begin{tabular}{|c|c|c|c|c|c|}
\hline Década & 60 & 70 & 80 & 90 & 2000 \\
\hline Algodón & $\mathrm{b}$ & $\mathrm{a}$ & $\mathrm{b}$ & $\mathrm{b}$ & $\mathrm{c}$ \\
\hline Arroz & $\mathrm{b}$ & $\mathrm{b}$ & $\mathrm{a}$ & $\mathrm{a}$ & $\mathrm{a}$ \\
\hline Trigo & $\mathrm{a}$ & $\mathrm{b}$ & $\mathrm{b}$ & $\mathrm{bc}$ & $\mathrm{c}$ \\
\hline Sorgo & $\mathrm{c}$ & $\mathrm{b}$ & $\mathrm{a}$ & $\mathrm{b}$ & $\mathrm{c}$ \\
\hline Maíz & $\mathrm{a}$ & $\mathrm{b}$ & $\mathrm{b}$ & $\mathrm{b}$ & $\mathrm{b}$ \\
\hline Caña de azúcar & bc & $\mathrm{c}$ & $\mathrm{b}$ & $\mathrm{a}$ & $\mathrm{a}$ \\
\hline Fríjol & d & $\mathrm{c}$ & b & $\mathrm{a}$ & b \\
\hline Yuca & $\mathrm{c}$ & $\mathrm{a}$ & $\mathrm{b}$ & $\mathrm{b}$ & $\mathrm{b}$ \\
\hline Café & $\mathrm{bc}$ & $\mathrm{b}$ & $\mathrm{a}$ & $a b$ & $\mathrm{c}$ \\
\hline Cacao & $\mathrm{d}$ & $\mathrm{c}$ & $\mathrm{b}$ & $\mathrm{a}$ & $b$ \\
\hline Hortalizas & d & $\mathrm{c}$ & $\mathrm{b}$ & $\mathrm{b}$ & $\mathrm{a}$ \\
\hline Papa & $\mathrm{c}$ & $\mathrm{b}$ & $\mathrm{a}$ & $\mathrm{a}$ & $\mathrm{b}$ \\
\hline Plátano & $\mathrm{c}$ & $\mathrm{b}$ & $a b$ & $a b$ & $\mathrm{a}$ \\
\hline Frutas & e & d & $\mathrm{c}$ & $\mathrm{b}$ & $\mathrm{a}$ \\
\hline Cereales & $a b$ & $a b$ & $\mathrm{a}$ & $\mathrm{a}$ & $\mathrm{b}$ \\
\hline Legumbres & $\mathrm{bc}$ & $a b$ & $\mathrm{a}$ & $a b$ & $\mathrm{c}$ \\
\hline \multicolumn{6}{|c|}{ Análisis multivariado de la varianza (MANOVA) } \\
\hline Wilks' Lambda & $<0,0001$ & $<0,0001$ & $<0,0001$ & $<0,0001$ & $<0,0001$ \\
\hline Pillai's Trace & $<0,0001$ & $<0,0001$ & $<0,0001$ & $<0,0001$ & $<0,0001$ \\
\hline Hotelling-Lawley & $<0,0001$ & $<0,0001$ & $<0,0001$ & $<0,0001$ & $<0,0001$ \\
\hline Roy's Greatest R & $<0,0001$ & $<0,0001$ & $<0,0001$ & $<0,0001$ & $<0,0001$ \\
\hline
\end{tabular}

El contraste canónico por su parte, como se observa en la tabla 6, permitió detectar diferencias entre la década de los 90 respecto a la del 70, 80 y la primera década del dos mil.

\begin{tabular}{cc}
\hline Década & Contraste \\
\hline 60 & $\mathrm{BA}$ \\
70 & $\mathrm{~B}$ \\
80 & $\mathrm{~B}$ \\
90 & $\mathrm{~A}$ \\
2000 & $\mathrm{~B}$ \\
\hline
\end{tabular}

El análisis de correlación y su valor de significancia, en la superficie cultivada para los principales cultivos entre 1961 y 2010, como se observa en la tabla 7, arrojó una relación directa entre el cultivo del algodón y trigo, es decir a menor superficie dedicada a la producción de algodón también se ha dado menor superficie dedicada para el cultivo del trigo. Se detectó una relación inversa y altamente significativa $(p<0.0001)$ entre el cultivo de algodón y de azúcar. De igual manera entre arroz y trigo. 
Tabla 7. Análisis de correlación superficie cultivada

\begin{tabular}{|c|c|c|c|c|c|c|c|c|c|}
\hline & & Algodón & Arroz & Trigo & Sorgo & Maíz & Caña de azúcar & Fríjol & Yuca \\
\hline Algodón & & 100.000 & $\begin{array}{c}-0.43608 \\
(0.0017)\end{array}$ & $\begin{array}{l}0.46537 \\
(0.0008)\end{array}$ & $\begin{array}{l}0.36502 \\
(0.0099)\end{array}$ & $\begin{array}{l}0.32404 \\
(0.0231)\end{array}$ & $\begin{array}{l}0.74589 \\
(<.0001)\end{array}$ & $\begin{array}{l}-0.26956 \\
(0.0611)\end{array}$ & $\begin{array}{l}0.30376 \\
(0.0338)\end{array}$ \\
\hline Arroz & & $\begin{array}{c}-0.43608 \\
(0.0017)\end{array}$ & 100.000 & $\begin{array}{l}-0.56745 \\
(<.0001)\end{array}$ & $\begin{array}{l}0.35710 \\
(0.0118)\end{array}$ & $\begin{array}{r}-0.22571 \\
(0.1189)\end{array}$ & $\begin{array}{l}0.50622 \\
(0.0002)\end{array}$ & $\begin{array}{l}0.58104 \\
(<.0001)\end{array}$ & $\begin{array}{l}0.25145 \\
(0.0814)\end{array}$ \\
\hline Trigo & & $\begin{array}{l}0.46537 \\
(0.0008)\end{array}$ & $\begin{array}{l}-0.56745 \\
(<.0001)\end{array}$ & 100.000 & $\begin{array}{l}-0.03643 \\
(0.8037)\end{array}$ & $\begin{array}{l}0.70092 \\
(<.0001)\end{array}$ & $\begin{array}{l}-0.63224 \\
(<.0001)\end{array}$ & $\begin{array}{l}-0.45771 \\
(0.0009)\end{array}$ & $\begin{array}{l}-0.28636 \\
(0.0461)\end{array}$ \\
\hline Sorgo & & $\begin{array}{l}0.36502 \\
(0.0099)\end{array}$ & $\begin{array}{l}0.35710 \\
(0.0118)\end{array}$ & $\begin{array}{c}-0.03643 \\
(0.8037)\end{array}$ & 100.000 & $\begin{array}{l}-0.05454 \\
(0.7097)\end{array}$ & $\begin{array}{l}-0.13276 \\
(0.3632)\end{array}$ & $\begin{array}{c}0.44739 \\
(0.0013)\end{array}$ & $\begin{array}{l}0.46251 \\
(0.0008)\end{array}$ \\
\hline Maíz & & $\begin{array}{l}0.32404 \\
(0.0231)\end{array}$ & $\begin{array}{l}-0.22571 \\
(0.1189)\end{array}$ & $\begin{array}{l}0.70092 \\
(<.0001)\end{array}$ & $\begin{array}{l}-0.05454 \\
(0.7097)\end{array}$ & 100.000 & $\begin{array}{l}-0.40898 \\
(0.0035)\end{array}$ & $\begin{array}{l}-0.20630 \\
(0.1550)\end{array}$ & $\begin{array}{l}-0.30687 \\
(0.0320)\end{array}$ \\
\hline $\begin{array}{l}\text { Caña } \\
\text { Azúcar }\end{array}$ & de & $\begin{array}{l}-0.74589 \\
(<.0001)\end{array}$ & $\begin{array}{l}0.50622 \\
(0.0002)\end{array}$ & $\begin{array}{l}-0.63224 \\
(<.0001)\end{array}$ & $\begin{array}{c}-0.13276 \\
(0.3632)\end{array}$ & $\begin{array}{c}-0.40898 \\
(0.0035)\end{array}$ & 100.000 & $\begin{array}{l}0.61595 \\
(<.0001)\end{array}$ & $\begin{array}{l}-0.11369 \\
(0.4367)\end{array}$ \\
\hline Fríjol & & $\begin{array}{c}-0.26956 \\
(0.0611)\end{array}$ & $\begin{array}{l}0.58104 \\
(<.0001)\end{array}$ & $\begin{array}{l}-0.45771 \\
(0.0009)\end{array}$ & $\begin{array}{l}0.44739 \\
(0.0013)\end{array}$ & $\begin{array}{c}-0.20630 \\
(0.1550)\end{array}$ & $\begin{array}{l}0.61595 \\
(<.0001)\end{array}$ & 100.000 & $\begin{array}{l}0.27935 \\
(0.0519)\end{array}$ \\
\hline Yuca & & $\begin{array}{l}0.30376 \\
(0.0338)\end{array}$ & $\begin{array}{l}0.25145 \\
(0.0814)\end{array}$ & $\begin{array}{l}-0.28636 \\
(0.0461)\end{array}$ & $\begin{array}{l}0.46251 \\
(0.0008)\end{array}$ & $\begin{array}{r}-0.30687 \\
(0.0320)\end{array}$ & $\begin{array}{l}-0.11369 \\
(0.4367)\end{array}$ & $\begin{array}{l}0.27935 \\
(0.0519)\end{array}$ & 100.000 \\
\hline
\end{tabular}

\section{Discusión de resultados}

Los resultados de este estudio indican que el uso de la tierra en Colombia ha tenido cambios marcados en las últimas décadas, con una reducción del área destinada a la producción agrícola. El contraste canónico indica que la mayor reducción se presentó en la década del 90; situación que coincide con la implementación en el país de la política de apertura económica, en la cual se cambió el modelo de desarrollo basado en el proteccionismo y altos subsidios, lo cual de acuerdo con Hernández (2009), implica un claro beneficio para los consumidores de bajos ingresos, en perjuicio del desarrollo agrícola interno y la soberanía alimentaria. Otros autores han encontrado a su vez que la reducción de aranceles a los alimentos, sin una reducción igual para los insumos y la maquinaria, no favorecen el crecimiento del sector agrícola (Ghezán, Mateos y Everdin, 2001).

Esta situación puede explicarse además por factores como el desplazamiento forzado consecuencia del conflicto armado que se recrudeció en el país a partir de la década del 90 (PNUD, 2011); situaciones que condujeron al estado a incrementar el gasto público para la defensa en detrimento del gasto público en las zonas rurales, lo cual de acuerdo con los hallazgos de autores como Kjöllerström (2004), tiene incidencia negativa en los indicadores de desarrollo agrícola. Esta misma situación produjo en el país a partir del 90 , el desmantelamiento de la institucionalidad para el desarrollo agropecuario, otro factor que puede explicar la reducción en el área destinada a la agricultura, por la disminución en el apoyo al pequeño productor en aspectos tan importantes como la asistencia técnica y el crédito, reportados por la literatura como claves para la innovación agrícola (Uaiene, Arndt, y Masters, 2009)

Los resultados del análisis multivariado de la varianza a su vez, indican una variación drástica en la superficie cultivada para los cultivos evaluados, lo cual representa un cambio 
estructural en la producción agrícola nacional en los últimos 50 años. Los resultados del análisis factorial indican la relación existente entre los cultivos de mayor crecimiento, caña de azúcar, frutales y hortalizas; el análisis de correlación y su valor de significancia, por su parte indica la relación directa existente entre algodón y trigo, los de mayor decrecimiento.

Con relación a loa anterior, se evidencia un efecto de las políticas públicas para el sector agrícola implementadas por los gobiernos nacionales en el período analizado, las cuales se orientaron al incremento de la productividad agrícola en el período 1970-1974; a la preocupación por la tenencia de la tierra, la productividad y el estímulo a la agricultura comercial en el período 1974-1978, a las cuales se les dio continuidad en el período 1978-1982; la oferta abundante de alimentos a bajo a costo y el crecimiento de las exportaciones en el período 1982-1986; la modernización del sector agropecuario y la búsqueda de la autosuficiencia alimentaria en el período 1986-1990; la liberalización del comercio internacional, el desmonte de controles a las importaciones y los precios de sustentación en el período 1990-1994, a las cuales se dio continuidad en el período 1994-1998 (Lorente, 1989; Jaramillo, 2002).

Por otra parte, los resultados del análisis de factor muestran como el factor uno se explicó por los cultivos que presentaron mayor crecimiento en la superficie sembrada en el período analizado: arroz, caña de azúcar, frijol, cacao, hortalizas, papa, plátano y frutas. Uno de los casos destacados de crecimiento en la superficie sembrada es el frijol, uno de los cultivos que más se utiliza en la dieta, principalmente en las zonas centro y cafetera del país. Adicionalmente, se destacó la tendencia creciente del cultivo de frutales, debido a la ampliación de las exportaciones y al incremento en el consumo interno, al igual que la creación y ampliación de factorías dedicadas a la elaboración de bebidas, lácteos y otros derivados, que utilizan la fruta como elemento principal.

Estos resultados indican que la producción de alimentos tiene una dinámica importante de crecimiento en el país, lo cual constituye una perspectiva interesante para Colombia al considerar el crecimiento sin precedentes de la demanda mundial de alimentos (Vélez, Campos, Córdoba y Anzola, 2010). Al respecto, de acuerdo con la FAO Colombia se ubica en el puesto 25 entre 223 países con respecto al potencial de expansión del área agrícola con un estimado de 10 millones de hectáreas (CEPAL, FAO, IICA, 2011), por cuanto el país tiene uno de los índices de utilización de la tierra arable más bajo de América Latina con apenas el $0.8 \%$ del potencial de tierras arables (Vélez, Campos, Córdoba y Anzola, 2010). Adicionalmente, se encuentra un alto nivel de tierras no aprovechadas o utilizadas sin atender criterios de vocación productiva.

Por otro lado, se destaca el crecimiento de la superficie sembrada en cultivos industriales como la caña de azúcar, principalmente destinada a la producción de biocombustibles, lo cual representa riesgos para el desarrollo del sector rural por cuanto como lo plantean autores como Michelini (2013) y FIAN (2006), las políticas públicas orientadas a la promoción de la agroindustria de gran escala, incrementan la marginalización de los pequeños productores y no contribuyen a la reducción de la pobreza en el área rural. Por otro parte, de acuerdo con los reportes de Arias y Salas (2006), este modelo incide en la disminución del tamaño de la propiedad agrícola, la reducción en la productividad, el manejo inadecuado e intensivo del suelo y el agua, llevando al sector agrícola a una situación de pobreza estructural. Pisani y 
Franceschetti (2011) plantean además que las políticas públicas orientadas a la promoción de la agricultura de exportación deben ir acompañadas del desarrollo de clústeres, la planificación del desarrollo con enfoque territorial, el capital institucional y la formación del capital humano.

Lo anterior implica la necesidad de identificar y plantear políticas para el desarrollo agrícola que permitan aprovechar las potencialidades del sector agrícola colombiano y su contribución a la reducción de la pobreza rural y la inequidad frente a las zonas urbanas. En este sentido, la literatura reporta como factores que se deben tener en cuenta el mejoramiento de las condiciones de tenencia de la tierra (Barrientos y Castrillón, 2007; Deininger, 2004; Rodríguez, 2011), acceso a seguros y créditos (Rigg, 2006); sistemas de investigación agrícola y transferencia de tecnología (Kjöllerström, 2004); asistencia técnica, extensión rural, cadenas de generación de valor (MGAP, 2011); consolidación de redes de actores del desarrollo (Esparcia, 2014); el fortalecimiento del capital social (Fawaz y Vallejos, 2011); el desarrollo de capacidades locales para la acción colectiva y el capital político (McDonalds, Kirk-Brown, Frost, Van Dijk y Rainnie, 2013); uso sostenible de la tierra y el agua en la agricultura (Carreño, Frank y Viglizzoa, 2012) y acceso al mercado, el mejoramiento organizaciones y mejoramiento de la institucionalidad pública (Bejarano, 1998).

\section{Conclusiones}

La evaluación de la dinámica de la superficie agrícola sembrada en Colombia, permitió establecer el descenso en el período analizado, lo cual coincide con la implementación de la política de apertura económica y representa un cambio estructural en la producción agrícola nacional en los últimos 50 años. Los cultivos de mayor crecimiento en la superficie sembrada y que por tanto plantean importantes perspectivas para el desarrollo agrícola del país en los próximos años fueron hortalizas, papa, plátano, frutales y cacao, los cuales representan una contribución importante a la producción de alimentos, al igual que caña de azúcar como cultivo con una orientación a la producción de biocombustibles.

Lo anterior implica la necesidad de implementar políticas para el desarrollo agrícola, que permitan aprovechar las potencialidades del sector agrícola colombiano para aprovechar su potencial de convertirse en despensa para abastecer la creciente demanda internacional de alimentos, con los consecuentes efectos que tendría como estrategia para la reducción de la pobreza rural y la inequidad en el desarrollo urbano-rural. Con base en los resultados del análisis de la dinámica de los últimos 50 años, estas políticas deben estar orientadas a paliar los efectos que tuvieron en el desarrollo agrícola del país la apertura económica y el conflicto armado, fortaleciendo la inversión pública para mejorar la institucionalidad de apoyo a los productores en aspectos fundamentales para el desarrollo agrícola de acuerdo con lo planteado en la literatura científica como el acceso a seguros y créditos, asistencia técnica y extensión rural y la promoción de las organizaciones de productores para mejorar el acceso al mercado.

\section{Referencias bibliográficas}

Arias, J. y Salas, V. (2006). Las experiencias de negociación agrícola entre la Comunidad Andina de Naciones (CAN) y la Unión Europea (UE). Revista Intercambio VIII.

Barrientos, J. y Castrillón, G. (2007). Generación de empleo en el sector agrario colombiano. Agronomía Colombiana, 25(2), 383-395. 
Baudasse, T. y Calderón, C. (2009). Integración comercial del sector agrícola y desigualdad económica en los países en vías de desarrollo. Inv. Econ, 68(269), 37-72.

Bejarano, J. (1998). El desarrollo institucional y la política agrícola. IICA: Costa Rica.

Carreño, L, Frank, F. \& Viglizzoa, E. (2012). Tradeoffs between economic and ecosystem services in Argentina during 50 years of land-use change. Agriculture, Ecosystems and Environment, 154, 68 77.

Cazalis, M. (1980). Agricultural development. Revista de Estudios Agro-Sociales, 112, 21-37.

CEPAL, FAO, IICA (2011). Perspectivas de la agricultura y del desarrollo rural en las Américas: una mirada hacia América Latina y el Caribe 2011-2012. San José, C.R.: IICA. 182 pp.

Deininger, K. (2004). Land Policies and Land Reform. Washington: World Bank, 37 pp.

Delgadillo, N. y Chalita, L. (2013). La política agrícola en México durante el periodo 1995-2009: un análisis multivariado. Rev. Mex. Cienc. Agríc, 4(2), 307-313.

Esparcia, J. (2014). Innovation and networks in rural areas. An analysis from European innovative projects. Journal of Rural Studies, 34, 1-14.

Fawaz, J. y Vallejos, R. (2011). Calidad de vida, ocupación, participación y roles de género: un sistema de indicadores sociales de sostenibilidad rural (Chile). Cuad. Desarro. Rural., 8 (67), 45-68.
FIAN. (2006). La reforma agraria en Paraguay. Informe de la misión investigadora sobre el estado de la realización de la reforma agraria en tanto obligación de derechos humanos. Germany: FIAN International. 38 pp. Tomado de: http:// www2.ohchr.org/english/bodies/cescr/ docs/info-ngos/fianparaguay.pdf

Ghezán, G, Mateos, M. \& Everdín, J. (2001). Impact of policy of structural adjusting in agricultural and agroindustrial sectors: The Argentina Case. Serie Desarrollo Productivo No. 90. Santiago, Chile: Economic Commission for Latin America and the Caribbean.

Hernández, J. (2009). Evolución y resultados del sector agroalimentario en la V República. CDC, 26(72), 67-100.

Jaramillo, C. F. (2002). Crisis y transformación de la agricultura colombiana 1990-2000. Bogotá: FCE-Banco de la República.

Kjöllerström, M. (2004). Competitividad del sector agrícola y pobreza rural: el papel de los gastos públicos en América Latina. Desarrollo productivo series, No. 155. Santiago, Chile: Economic Commission for Latin America and the Caribbean (ECLAC).

Lorente, L. (1989). "La oferta selectiva de alimentos" en Coyuntura Agropecuaria No.21-22. Bogotá: CEGA.

Lowe, P, Murdoch, J, Marsden, T, Munton, R. \& Flynn, A. (1993). Regulating the new rural spaces: the uneven development of land. Journal of Rural Studies, 9 (3), 205-222. 
MGAP. (2011). Proyecto Uruguay Rural, aportes a la construcción del desarrollo rural: síntesis de resultados y lecciones aprendidas período 2005-2011. Uruguay: Ministerio de Ganadería, Agricultura y Pesca, Dirección General de Desarrollo Rural (DGDR), 127 pp. Recuperado el 28 de julio de 2014, de: http://www.mgap.gub.uy/portal/ agxppdwn.aspx?7,10,262,O,S,0,4875\% 3BS\%3B1\%3B156.

Michelini, J. (2013). Small farmers and social capital in development projects: Lessons from failures in Argentina's rural periphery. Journal of Rural Studies, 30, 99-109.

McDonald, C, Kirk-Brown, A, Frost, L, Van Dijk, P. \& Rainnie, A. (2013). Partnerships and integrated responses to rural decline: The role of collective efficacy and political capital in Northwest Tasmania, Australia. Journal of Rural Studies, 32, 346-356.

Norton, R. (2004). Política de desarrollo agrícola: conceptos y principios. Roma: FAO. $591 \mathrm{pp}$.

Pisani, E, y Franceschetti, G. (2010). Da agrariedade á ruralidade: Novos enfoques á economia territorial. Economía, 35(30), 79-101.

Porter, M. (2007). La ventaja competitiva de las naciones. Harvard Business Review, 4-23.

PNUD (2011). Colombia rural. Razones para la esperanza. Informe Nacional de Desarrollo Humano 2011. Bogotá: INDH PNUD
Restrepo, J. (2010). Una política integral de tierras para Colombia. Bogotá: Ministerio de Agricultura y Desarrollo Rural.

Rigg, J. (2006). Land, Farming, Livelihoods, and Poverty: Rethinking the Links in the Rural South. World Development, 34(1), 180-202.

Rodríguez, J.(2011). Vías de desarrollo, cambio tecnológico y políticas estructurales en la agricultura moderna venezolana. $C D C, 28(76), 67-96$.

Soto, C. (2003). La agricultura comercial de los distritos de riego en México y su impacto en el desarrollo agrícola. Invest. Geog, 50, 173-195.

Uaiene, R., Arndt, C, \& Masters, W. (2009). Determinants of agricultural technology adoption in Mozambique. Discussion Papers (67).Retrievedfromhttp://196.46.4.208/gest/ documents/67E_AgTechAdoptionMoz.pdf

Vélez, A, Campos, A, Córdoba, S. y Anzola, J. (2010). Sector Agroindustrial Colombiano. Invierta en Colombia, vol $1,1-10$. 